\title{
A comprehensive mental health care program for women with polycystic ovary syndrome: protocol for a mixed methods study
}

\author{
Fatemeh ZareMobini ${ }^{1}$, Ashraf Kazemi ${ }^{2}$ and Ziba Farajzadegan ${ }^{3^{*}}$
}

\begin{abstract}
Background: Psychological health is related to the management of polycystic ovary syndrome (PCOS) and is an essential component of self-efficacy and enjoying a healthy lifestyle. Need assessment and plans to improve the psychological health of these women provides significantly valuable information to design an advantageous intervention program to reach that goal. Therefore, this study will conduct to improve the psychological health for women with PCOS through a designed comprehensive care.

Methods: This is an exploratory mixed method study using consecutive qualitative-quantitative methods, including three consecutive phases. In the first phase, a qualitative study will be conducted to assess their needs and will design a care protocol for improving mental health of women with PCOS. Participants in this phase will select by purposive sampling method and data will collect using semi-structured interviews by taking notes at same time. Data will analyze using conventional content analysis method. At second phase, according the information obtained from previous phase and a literature review a comprehensive program to mental health care will be proposed. Then multidisciplinary team will review and finalize it according to priorities. The third phase will follow a quantitative approach using quasi-experimental study with two groups to measure the effectiveness of this program on the women's psychological health.

Discussion: Designing a program based on a qualitative study and a review article and updated evidences can lead to improving of these women's psychological health and quality of life. Consequently, we expect to show that mental health program provided by a multidisciplinary team improves reproductive outcomes while at the same time being cost-effective in women with PCOS.
\end{abstract}

Keywords: Intervention program, Psychological health, Polycystic ovary syndrome (PCOS), Needs, Reproductive health, Mental health promotion

\section{Plain English summary}

Psychological health is related to the management of polycystic ovary syndrome (PCOS) and is an essential component of self-efficacy and enjoying a healthy lifestyle. The present study provides strong information and data regarding the needs and strategies for improving the psychological health in women with PCOS. This is

\footnotetext{
* Correspondence: Z_farajzadegan1361@yahoo.com

${ }^{3}$ Community medicine Department, Faculty of medicine, Isfahan University of Medical Sciences, Isfahan, Iran

Full list of author information is available at the end of the article
}

an exploratory mixed method study using consecutive qualitative-quantitative methods, including three consecutive phases. In the first phase, a qualitative study will be conducted to assess their needs and will design a care protocol for improving mental health of women with PCOS. Participants in this phase will select by purposive sampling method and data will collect using semistructured interviews by taking notes at same time. Data will analyze using conventional content analysis method. At second phase, according the information obtained from previous phase and a literature review a comprehensive 
program to mental health care will be proposed. Then multidisciplinary team will review and finalize it according to priorities. The third phase will follow a quantitative approach using quasi-experimental study with two groups to measure the effectiveness of this program on the women's psychological health. Therefore, designing a program based on a qualitative study and a review article and updated evidences can lead to improving of these women's psychological health and quality of life. Consequently, we expect to show that mental health program provided by a multidisciplinary team improves reproductive outcomes while at the same time being cost-effective in women with PCOS.

\section{Background}

Polycystic Ovarian Syndrome (PCOS), the most common endocrine disorder in women of reproductive age; its prevalence has been shown to be 6 to $18 \%$ based on Rotterdam diagnostic criteria [1-3]. Clinical presentation includes oligomenorrhea, obesity, acne, reproductive disorders and hirsutism [4-6]. Moreover, because of impaired glucose tolerance, insulin resistance, type II diabetes, an abnormal increase in blood lipids and high blood pressure, patients with PCOS are at increased risk of cardiovascular disease [7, 8]. Also, due to its chronic nature and variety of its symptoms, PCOS affect the patients' different aspects of life. Studies suggest that in addition to physical problems [8], and emotional disorders [9] their quality of life is impacted by disorder [10], and PCOS leads to psychological problems [9-18]. Pcos creates an important psychological burden throughout the life-time of these women $[9,10,19]$. It has been reported that $57 \%$ of women with polycystic ovary syndrome have at least one mental disorder [20]. High levels of androgens, acne, infertility and fear of it, hirsutism, high body mass index, low body image, sexual problems and coping with the disease impair the mental health of them [20,21].

Mental health is fundamental to good health and wellbeing and influences social and economic outcomes across the lifespan [22]. Also, mental health is an important part of public health [23] and it is one of the most important indicators of the quality of health care in society [24]. Many chronic illnesses have mental health impacts [25]. In PCOS, symptoms and comorbidities increase the risk of adverse psychological health consequences. While, mental health is particularly related to the management of polycystic ovary syndrome and is an essential component of self-efficacy and enjoying a healthy lifestyle $[20,25]$. Lifestyle modification is the priority in the management of polycystic ovary syndrome, since small changes in these women's lifestyle and a balanced weight, can improve symptoms, and increase ovulation and improve fertility $[6,26]$. However, mental disorders including depression or anxiety reduce these critical components and prevent of lifestyle modification and subsequently the disease will show negative clinical outcomes [25, 27]. Considering the importance of mental health, some researchers suggest that there has been little attention paid to the mental health of women with PCOS, and it is necessary to evaluate and manage the mental health among this group of women [28]. Therefore, this exploratory mixed method study will carry out to define and explore their needs and demands to provide a standard patient oriented protocol for women with PCOS.

\section{Methods}

This is an exploratory mixed method study using consecutive qualitative-quantitative methods, including three consecutive phases. In the first phase, a qualitative study will conduct and the conclusion resulted from this phase will analyze using a conventional content analysis method to determine the needs and strategies related to improving mental health among the women with PCOS.

At second phase, according the information obtained from previous phase and a literature review a comprehensive program to mental health care will be proposed. Then multidisciplinary team will review and finalize it according to priorities.

The third phase will follow a quantitative approach using quasi-experimental study with two groups to measure the effectiveness of this program on the women's psychological health. The general design of the study is presented in Fig. 1.

\section{Phase I: Qualitative study}

In this phase of the study, needs and strategies related to the mental health of women with PCOS will determine using semi-structured deep interviews, which will analyze using conventional content analysis method. The study environment included women's health clinics, clinics for endocrinology, dermatology, laser centers, and infertility centers affiliated to the Isfahan University of Medical Sciences. All interviews will conduct in a private and contented place. Health worker interview will be held in their office.

\section{Participants}

The participants of this study include women with PCOS based on Rotterdam diagnostic criteria with the maximum diversity of the disease range, duration of their illness, age, education, marital status, social class, job and their spouses. Also, health service providers with an experience in providing health care and treatment services to women with PCOS including gynecologists, dermatologists, endocrinologists, nutritionist, reproductive health specialists, general practitioners, midwives, psychiatrists, psychologists, social workers also will participate in 


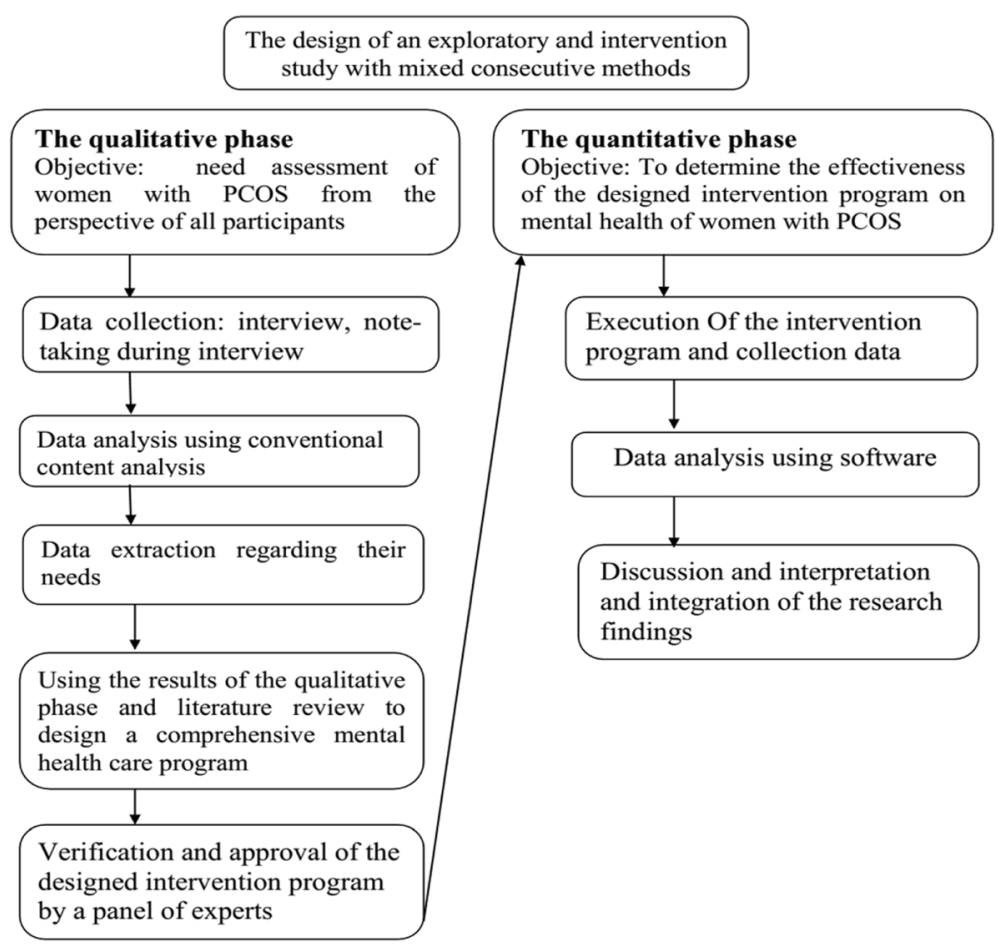

Fig. 1 The general design of the study

the study with informed consent. Participants will select through purposive sampling, and the interviews will conduct with those who had the inclusion criteria after receiving their informed consent. We continue the interviews until data saturation, and the interviews will stop if data saturation occurs.

\section{Inclusion criteria}

Iranian women with a confirmed diagnosis of PCOS, who have competency to take, participate in interview, no history of cancer or chronic and psychological disorder. Health care providers with at least one year experience for caring this women.

\section{Qualitative data collection method}

In this phase, data will gather through open and semi-structured interviews as well as taking notes on the spot. The following actions will be performed for ethical consideration, this study was confirmed by the Ethical Committee of Isfahan University of Medical Sciences, explanations will provided for the participants regarding the objectives of the study. Written and verbal consent will obtain from the participants for recording their interviews. Time schedule, length and place of interview will selected with participant preference.
The method for qualitative data analysis

Conventional content analysis will apply for data analysis and interpretation.

\section{Accuracy and reliability of the qualitative data}

To assure the trustworthiness of the findings, the four criteria credibility, dependability, confirmability, transferability will apply [29].

\section{Phase II: Designing the intervention protocol}

In the second phase of the study, a comprehensive mental health care program will prepared based on need assessment and approaches extracted from the qualitative study and literature review to improve the mental health of women with PCOS, and it will validate by an experts panel. Literature review will conduct using narrative review method included searching library sources (reference books, thesis, and dissertations) as well as electronic database search to access the existing knowledge related to issue. In this phase, all studies with key words including; PCOS, AND "mental health" plus PCOS, AND "mental health improvement" that were published in English or Persian, between 2006 and 2017 will review. Databases of PubMed, ScienceDrect, Web of Science, Cochrane Library, Ovid, Scopus, ProQuest, Magiran, Embase, SID Database Will search. 


\section{Holding an multidisciplinary team}

At this step, to achieve experts' consensus, the technique of RAM (Rand Appropriateness Method) will use [30]. The process is as follow: using the results of the qualitative study and reviewing the literature, creating and sending a list of health care services to multidisciplinary team. The multidisciplinary team will grade each health care service considering benefits to harm ratio on the scale of 1 to 9 , where 1 means the expected harms are higher than the benefits, and 9 means its expected benefits greatly outweigh the harm. Finally, each item in the list will classify as "Good," "indeterminate" or "not good" based on the ratings and scores. In the second round, a meeting of all multidisciplinary team will be held, including experts in endocrinology, reproductive health, dermatology, nutritionist, obstetrics and gynecology, midwives, psychologists and psychiatric nurses. During the meeting, these experts discuss the prioritization of strategies while taking into account the conditions and the time limitation for the intervention process.

\section{Phase III: Quantitative study}

In this step a randomized controlled clinical trial will design to assess the efficacy of prepared protocol. Participants randomly will allocate into two groups. In experimental group we will implement our designed protocol, and control group will not receive any intervention, but they follow as same as experimental group. For ethical issues all educational material will offer them at the end of study. Mental health status will assess as a desired outcome.

\section{Study environment and population}

Ambulatory clinics for gynecology, endocrinology, dermatology, laser therapy centers, and infertility centers affiliated to the Isfahan University of Medical Sciences will consider as a research environment.

\section{Study sample}

Target populations for study are all women with confirmed diagnosis of PCOS based on Rotterdam diagnostic criteria.

\section{Sampling method}

Convenience sampling's method will apply to select the participants.

\section{Inclusion criteria}

Inclusion criteria are women aged between 15 and 49 years, Iranian nationality, consent to participate in the study, ability to understand questions or ability to read and write, not being in stressful situations such as immigration, death of loved ones and financial problems in the past 6 months, not participating in any other clinical trials at the same time, no severe mental disorders that requires to be under medical treatment or hospitalization.

\section{Exclusion criteria}

The exclusion criterion is not completing the intervention for any reason such as severe life stress, the death of the spouse or immigration and so on.

\section{Data collection method}

The instrument for collecting mental health data is the GHQ-28.This questionnaire has 4 subscales including somatic symptoms, anxiety/insomnia, social dysfunction, and severe depression. The cut of point of the questionnaire is 23; which means that a score less than 23 signifies mental health and 23 and above signifies mental health problem and the subject needs to be referred to a psychiatric.

\section{Data analysis:}

We will apply $\mathrm{X}^{2}$ and independent $\mathrm{T}$ test to analysis our data by using SPSS version 21 .

\section{Discussion}

Various studies emphasized on the necessity of mental health evaluation and management among women with PCOS $[5,28,31-33]$ and suggested that addressing the mental health problems should be a routine part of the health evaluation in clinical setting [14, 16, 28, 33, 34]. Also, studies explain that since there has been little attention paid to the psychological effects of this disease, it is necessary to plan for comprehensive care, including psychological aspects of PCOS and determine the needs of women with this disease [31,34]. The present study provides strong information and data regarding the needs and strategies for improving the mental health in women with PCOS. Therefore, designing a program based on a qualitative study and by considering the context, and a review article and updated evidences can lead to enhancement of these women's mental health and quality of life. It can also reduce their medical and treatment costs. We suppose this program has capacity to integrate into the professionally health care guidelines, so that it can help medical and health care providers pay attention to the important role of the mental health alongside treatment methods of the physical and mental problems of women with PCOS, especially during reproductive age. The strategies of this program could be important and cost effective, and therefore we hope that the success of such a program is a step forward in improving their health status and quality of life. 


\section{Abbreviations}

GHQ-28: General Health Questionnaire-28; PCOS: Polycystic Ovarian Syndrome; RAM: Rand Appropriateness Method

\section{Acknowledgments}

This paper was extracted from a reproductive health PhD thesis. The authors would like to thank Isfahan University of Medical Sciences for supporting this research.

\section{Funding}

This research protocol was funded by the Isfahan University of Medical Sciences, Isfahan, Iran (Grant no. 395508).

\section{Availability of data and materials} Not applicable.

\section{Authors' contributions}

FZM, AK, ZF were involved in study conception, design and drafting of the manuscript. FZM wrote the first draft of this study protocol. AK and ZF reviewed the first draft of the protocol and manuscript. AK provided the qualitative design. ZF and AK were responsible for coordinating the study. FZM will be responsible for interview with participants, description and data analysis. AK will review and will involve in data analysis and qualitative phase. FZM will be responsible for delivering and intervention. ZF will provide the quantitative design. All authors have read and approved the final version of the manuscript.

\section{Ethics approval and consent to participate}

Ethical approval for this study has been obtained by the ethics committee affiliated with Isfahan University of Medical Sciences, Isfahan, Iran (IR.MUI.REC. 1395.3.508). Registration of this randomized control trial has been completed with the Iranian Registry of Clinical Trials, IRCT2017092736445N1.

\section{Consent for publication}

Not applicable.

\section{Competing interests}

The authors declare that they have no competing interests.

\section{Publisher's Note}

Springer Nature remains neutral with regard to jurisdictional claims in published maps and institutional affiliations.

\section{Author details}

${ }^{1}$ Student Research Center, Faculty of Nursing and Midwifery, Isfahan University of Medical Sciences, Isfahan, Iran. ${ }^{2}$ Reproductive health Department, Faculty of Nursing and Midwifery, Isfahan University of Medical Sciences, Isfahan, Iran. ${ }^{3}$ Community medicine Department, Faculty of medicine, Isfahan University of Medical Sciences, Isfahan, Iran.

\section{Received: 22 October 2017 Accepted: 6 March 2018}

Published online: 13 March 2018

\section{References}

1. Blagojevic IP, Eror T, Pelivanovic J, Jelic S, Kotur-Stevuljevic J, Ignjatovic S. Women with polycystic ovary syndrome and risk of cardiovascular disease. J Med Biochem. 2017;36:259-69.

2. Raja-Khan N, Agito K, Shah J, Stetter CHM, Gustafson TS, Socolow H, et al. Mindfulness-based stress reduction for overweight/obese women with and without polycystic ovary syndrome: design and methods of a pilot randomized controlled trial. Contemp Clin Trials. 2015;41:287-97.

3. Conte F, Banting L, Teede HJ, Stepto NK. Mental health and physical activity in women with polycystic ovary syndrome: a brief review. Sports Med. 2015:45:497-504.

4. Rees DA, S J-J, Morgan CL. Contemporary reproductive outcomes for patients with polycystic ovary syndrome: a retrospective observational study. J Clin Endocrinol Metab. 2016;101(4):1664-72.

5. Hart R, Doherty DA. The potential implications of a PCOS diagnosis on a woman's long-term health using data linkage. J Clin Endocrinol Metab. 2015;100(3):911-9.
6. Palomba S, Santagni S, Falbo A, Battista G. Complications and challenges associated with polycystic ovary syndrome: current perspectives. Int J Women's Health. 2015;7:745-63.

7. Aziz M, Sidelmann JJ, Faber J, Wissing ML, Naver KV, Mikkelsen AL, et al. Polycystic ovary syndrome: cardiovascular risk factors according to specific phenotypes. Acta Obstet Gynecol Scand. 2015:94(10):1082-9.

8. Balen AH. Polycystic ovary syndrome (PCOS). The Obstetrician \& Gynaecologist. 2017;19:119-29.

9. Teede H, Deeks A, Moran L. Polycystic ovary syndrome: a complex condition with psychological, reproductive and metabolic manifestations that impacts on health across the lifespan. BMC Med. 2010;8:41-51.

10. Y L, Li Y, Yu Ng EH, Stener-Victorin E, Hou L, Wu T. Polycystic ovary syndrome is associated with negatively variable impacts on domains of health-related quality of life: evidence from a meta-analysis. Fertil Steril. 2011:96(2):452-8.

11. Scaruffi E, Gambineri A, Cattaneo S, Turra J, Vettor R, Mioni R. Personality and psychiatric disorders in women affected by polycystic ovary syndrome. Front Endocrinol (Lausanne). 2014;5:185

12. Tan J, Wang QY, Feng GM, Li XY, Huang W. Increased risk of psychiatric disorders in women with polycystic ovary syndrome in Southwest China. Chin Med J. 2017;130:262-6.

13. Himelein $M$, Thatcher S. Polycystic ovary syndrome and mental health: a review. Obstetrical \& Gynecological Survey. 2006:61:723-32.

14. Kerchner A, Lester W, Stuart S, Dokras A. Risk of depression and other mental health disorders in women with polycystic ovary syndrome: a longitudinal study. Fertil Steril. 2009;91:207-12.

15. Rachel N. PCOS T.A.C.T.: a program to assist psychologists in understanding and helping women diagnosed with polycystic ovary syndrome. The Chicago school of Prof Psychol. 2012;154

16. Hung $\mathrm{JH}$, et al. Risk of psychiatric disorders following polycystic ovary syndrome: a Nationwide population-based cohort study. PLoS One. 2014:9(5):e97041.

17. Asik M, Altinbas K, Eroglu M, Karaahmet E, Erbag G, Ertekin H, et al. Evaluation of affective temperament and anxiety-depression levels of patients with polycystic ovary syndrome. J Affect Disord 2015, 1. 185:214-8.

18. Annagür BB, Kerimoglu ÖS, Tazegül A, Gündüz \$̧, Gençoglu BB. Psychiatric comorbidity in women with polycystic ovary syndrome. J Obstet Gynaecol Res. 2015:41(8):1229-33.

19. Stefanaki C, Bacopoulou F, Livadas S, Kandaraki A, Karachalios A, Chrousos $\mathrm{GP}$, et al. Impact of a mindfulness stress management program on stress, anxiety, depression and quality of life in women with polycystic ovary syndrome: a randomized controlled trial. Stress. 2015;18(1):57-66.

20. Rodrigues CEG, Ferreira L, Jansen K, Lopez M. Evaluation of common mental disorders in women with polycystic ovary syndrome and its relationship with body mass index. Rev Bras Ginecol Obstet. 2012;34:442-6.

21. Podfigurna-Stopa A, Luisi S, Regini C, Katulski K, Centini G, Meczekalski B, et al. Mood disorders and quality of life in polycystic ovary syndrome. Gynecol Endocrinol. 2015;31(6):431-4

22. Barry MM, Clarke AM, Jenkins $R$, Patel V. A systematic review of the effectiveness of mental health promotion interventions for young people in low and middle income countries. BMC Public Health. 2013;13:835.

23. Molarius A. Mental health symptoms in relation to socio-economic conditions and lifestyle factors - a population-based study in Sweden. BMC Public Health. 2009;9:302.

24. Whiteford HA, Ferrari AJ, Degenhardt L, Feigin V, Vos T. The global burden of mental, neurological and substance use disorders: an analysis from the global burden of disease study 2010. PLoS One. 2015;10(2):1-14.

25. Banting LK, Gibson-Helm M, Polman R, Teede HJ, Stepto NK. Physical activity and mental health in women with polycystic ovary syndrome. BMC Womens Health. 2014;14:51.

26. Domecq JP, Prutsky G, Mullan RJ, Hazem A, Sundaresh V, Elamin MB, et al. Lifestyle modification programs in polycystic ovary syndrome: systematic review and meta-analysis. J Clin Endocrinol Metab. 2013;98(12):4655-63.

27. Deeks AA, Gibson-Helm ME, Teede HJ. Anxiety and depression in polycystic ovary syndrome: a comprehensive investigation. Fertil Steril. 2010;93:2421-3.

28. Moran LJ, March WA, Whitrow MJ, Giles LC, Davies MJ, Moore VM. Sleep disturbances in a community-based sample of women with polycystic ovary syndrome. Hum Reprod. 2015;30(2):466-72.

29. Streubert HJ, Carpenter DR. Qualitative research in nursing. Fifth ed. Philadelphia: Lippincott Williams \& Wilkins; 2011 
30. Fitch K, Bernstein SJ, Aguilar MS, Burnand B, Lacalle JR, et al. Rand appropriateness method user,s manual. Santa monica, CA: RAND Corporation; 2001.

31. Farrell-Turner KA. Women's health providers: don't forget about polycystic ovary syndrome. Am Psychol. 2015;70(1):49-50.

32. Lara LA, Ramos FK, Kogure GS, Costa RS, Silva de Sá MF, Ferriani RA, et al. Impact of physical resistance training on the sexual function of women with polycystic ovary syndrome. J Sex Med. 2015;12(7):1584-90.

33. Greenwood EA, Pasch LA, Shinkai K, Cedars MI, Huddleston HG. Putative role for insulin resistance in depression risk in polycystic ovary syndrome. Fertil Steril. 2015;104(3):707-14.

34. Nasiri Amiri F, Ramezani Tehrani F, Simbar M, Montazeri A, Mohammadpour RA. The experience of women affected by polycystic ovary syndrome: a qualitative study from Iran. Int J Endocrinol Metab. 2014;12(2):e13612.

Submit your next manuscript to BioMed Central and we will help you at every step:

- We accept pre-submission inquiries

- Our selector tool helps you to find the most relevant journal

- We provide round the clock customer support

- Convenient online submission

- Thorough peer review

- Inclusion in PubMed and all major indexing services

- Maximum visibility for your research

Submit your manuscript at www.biomedcentral.com/submit 